OPEN ACCESS

Edited by:

Santi M. Mandal,

Indian Institute of Technology

Kharagpur, India

Reviewed by:

Tridib Kumar Sinha,

Gyeongsang National University,

South Korea

César de la Fuente,

Massachusetts Institute of

Technology, United States

*Correspondence:

Fengming Lin

linfengming@seu.edu.cn

Zhan Chen

zhanc@umich.edu

Specialty section:

This article was submitted to Antimicrobials, Resistance and

Chemotherapy,

a section of the journal

Frontiers in Microbiology

Received: 14 November 2017 Accepted: 01 February 2018

Published: 16 February 2018

Citation:

Lin F, Li C and Chen Z (2018) Bacteria-Derived Carbon Dots Inhibit Biofilm Formation of Escherichia coli without Affecting Cell Growth

Front. Microbiol. 9:259. doi: 10.3389/fmicb.2018.00259

\section{Bacteria-Derived Carbon Dots Inhibit Biofilm Formation of Escherichia coli without Affecting Cell Growth}

\author{
Fengming Lin*, Chengcheng Li and Zhan Chen* \\ State Key Laboratory of Bioelectronics, School of Biological Science and Medical Engineering, Southeast University, Nanjing, \\ China
}

Biofilms are deleterious in many biomedical and industrial applications and prevention of their formation has been a pressing challenge. Here, carbon dots, CDs-LP that were easily synthesized from the biomass of Lactobacillus plantarum by one-step hydrothermal carbonization, were demonstrated to prevent biofilm formation of $E$. coli. CDs-LP did not thwart the growth of $E$. coli, indicating the anti-biofilm effect was not due to the bactericidal effect. Moreover, CDs-LP did not affect the growth of the animal cell AT II, showing low cytotoxicity, good safety and excellent biocompatibility. Therefore, CDs-LP could overcome the cytotoxicity issue found in many current antibiofilm agents. CDs-LP represent a new type of anti-biofilm materials, opening up a novel avenue to the development of biofilm treatment.

Keywords: biofilm, carbon dots, lactic acid bacterium, biocompatibility, antifouling

\section{INTRODUCTION}

Biofilms are highly structured communities of immobile microorganisms that are adhered to a surface or to each other, and embedded in a protective extracellular polymeric matrix consisting of polysaccharides, nucleic acids, proteins, and lipids (Flemming et al., 2016). Biofilms are frequently found on natural, clinical and industrial settings, causing tremendous issues due to the fact that they are resistant to external stresses, host defenses and conventional antimicrobial agents (de la Fuente-Núñez et al., 2013). For example, biofilms represent major virulence factors, resulting in chronic human infections, implant failure and even death (Anderson et al., 2003; Duncan et al., 2015). Also, biofilms are harmful for industrial facilities by blocking filtration membranes, fouling marine surfaces, or corroding pipes (Natalio et al., 2012). However, combating these biofilms, by either prevention or eradication, remains particularly challenging in both industrial and biomedical applications.

Annihilation of biofilm in vivo with traditional therapeutic antibiotics is becoming an overwhelming task. Antimicrobial therapy alone is rarely effective, and surgical intervention is often needed, resulting in a prolonged course of medical treatment with high costs (Kostakioti et al., 2013). Even worse, the development of new antibiotics is slow and difficult, which cannot keep pace with the emergence of antibiotic-resistant microorganisms (Fischbach and Walsh, 2009). Thus, alternative strategies to combat biofilm are highly desired. With the rapid development of nanotechnology, an increasing number of nanomaterials with unique properties have been explored for fighting various biofilms. Such nanomaterials include metal/metal oxide nanoparticles (Taglietti et al., 2014; Boda et al., 2015; Nguyen et al., 2015; Geilich et al., 2017; Hu et al., 2017) along with polymeric nanoparticles (Duong et al., 2014; Nguyen et al., 2016), nanoparticle-stabilized 
capsules (Duncan et al., 2015), nanoenzymes (Natalio et al., 2012; Chen et al., 2016), hydrogels(Gao et al., 2014), liposomes (Robinson et al., 2001) and so on, in either antibiotic-free or antibiotic-coated ways. A big concern with the application of nanomaterials in killing biofilm is cytotoxicity which was also often observed in many conventional antibiofilm materials. Despite their excellent anti-biofilm activity, nanomaterials are usually toxic toward microorganisms and human cells, and are consequently not biocompatible ( $\mathrm{Hu}$ et al., 2017). This non-biocompatibility issue caused safety concerns, decreased therapeutic selectivity, and adverse effects on ecosystems, and thus needs to address urgently. To solve this problem, surface engineering is routinely explored to finely tune nanomaterials' surface properties to achieve concurrent antibiofilm activity and non-cytotoxicity, which nevertheless involves the use of complex chemical/physical modification methods (Giri et al., 2015; Hu et al., 2017).

In this study, we present a novel type of carbon dots, easily prepared using $L$. plantarum as a single carbon source by one-step hydrothermal reaction without any chemical/physical modification, to prevent $E$. coli biofilm formation with excellent biocompatibility (Scheme 1). Carbon dots have wide applications in many fields including sensing, bio-imaging, photo-catalysis, drug delivery, etc., because of their facile synthesis route, exceptional photostability, low toxicity, great water dispersibility and amenable surface modification (Li et al., 2012; Wang and $\mathrm{Hu}, 2014)$. They have been successfully employed to discriminate gram negative/positive bacteria (Yang et al., 2016) as well as live/dead microorganisms (Hua et al., 2017b). The amphiphilic carbon dots have been synthesized and utilized for efficiently labeling biomimetic and cellular membranes (Nandi et al., 2014, 2015). Furthermore, carbon dots usually possess varied surface functional groups and can be easily engineered by other drugs and ligands for the applications in organelletargeted imaging and/or drug delivery (Yang et al., 2015; Zhang et al., 2015; Hua et al., 2017a). For biofilm application, carbon dots have been successfully exploited to image biofilm matrix (Ritenberg et al., 2016) and biofilm-encased microorganisms (Lin et al., 2017). However, to the best of our knowledge, the utilization of carbon dots in combating biofilm has not been reported.

\section{MATERIALS AND METHODS}

\section{Preparation and Purification of CDs}

CDs-LP were made from $L$. plantarum LLC-605 by one-step hydrothermal carbonization. L. plantarum LLC-605 was isolated in our lab from the traditional Chinese fermented food FuYuan pickles (Yunnan, China), which was deposited in NCBI with the accession number KX443590 (Li et al., 2017). L. plantarum LLC-605 was cultured in $30 \mathrm{~mL}$ De Man, Rogosa and Sharpe (MRS) medium at $31^{\circ} \mathrm{C}$ for $18 \mathrm{~h}$ without shaking. The overnight cell culture was centrifuged at 5,000 rpm for $10 \mathrm{~min}$ to remove the supernatant, washed three times with Milli-Q water, and transferred into a $50 \mathrm{~mL}$ Teflon-lined stainless-steel autoclave, and re-suspended in $30 \mathrm{~mL}$ Milli-Q water. The autoclave was incubated at $200^{\circ} \mathrm{C}$ for $24 \mathrm{~h}$. To remove large particles/aggregates, the synthesized dark brown solution was centrifuged at 12,000 $\mathrm{rpm}$ for $10 \mathrm{~min}$, and the supernatant was filtered through a $0.22 \mu \mathrm{m}$ filter membrane. Then, the CD solution was dialyzed against Milli-Q water in a dialysis bag with molecular weight cut-off of $1 \mathrm{kDa}$ for 2 days, and stored at $4^{\circ} \mathrm{C}$ for further experiments. For quantification and long-term storage, the CDs were lyophilized and weighed after dialysis.

\section{Characterizations of CDs}

The morphology, size and element components of the assynthesized CDs-LP were determined. A drop of CDs-LP solution was deposited on a 400-mesh carbon-coated copper grid and examined by a transmission electron microscope (TEM, JEM-2100, JEOL Ltd., Japan). Ultraviolet-visible (UVvis) and fluorescence spectra of CDs-LP in pure water or phosphate-buffered saline (PBS: $137 \mathrm{mM} \mathrm{NaCl}, 2.7 \mathrm{mM} \mathrm{KCl}$,

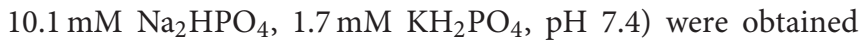
by a UV-vis spectrophotometer (UV-2600, Shimadzu, Japan) and a spectrofluorophotometer (RF-5301PC, Shimadzu, Japan), respectively. Fourier transform infrared (FTIR) spectroscopic experiment was carried out with an FTIR spectrometer (Nicolet iS50, Thermo Scientific, USA). X-ray photoelectron spectroscopic (XPS) experiment was performed via a Japan Kratos Axis Ultra HAS spectrometer. Zeta potential values of CDs-LP were determined with a Zetasizer instrument (Malvern Instruments, Nano ZS, United Kingdom). The fluorescence quantum yield (QY) of the CDs-LP was calculated according to the method previously reported (Yang et al., 2015).

\section{Inhibition of $E$. coli Biofilm Formation}

Bacteria were cultured overnight in $\mathrm{LB}$ at $37^{\circ} \mathrm{C}$ with $180 \mathrm{rpm}$. The overnight bacterial cell culture was diluted 1:100 in 1/5 LB without and with different concentrations of CDs-LP and then inoculated into the 96-well plates (Costar, Corning, USA) with $100 \mathrm{uL} /$ well or the glass bottom cell culture dish (NEST, USA) with $2 \mathrm{~mL} /$ dish. The inoculated 96-well plates and dishes were incubated for $1-5$ days at $28^{\circ} \mathrm{C}$ without shaking (Jurcisek et al., 2011; Duncan et al., 2015).

\section{Confocal Imaging}

For confocal imaging, static biofilm was cultured and processed inside glass bottom culture dishes (Nest, USA) following the methods as described above. The grown biofilms were washed with PBS three times, stained with a live/dead assay (Filmtracer LIVE/DEAD Biofilm Viability Kit, Thermo Fisher Scientific) and then examined under a confocal microscope (TCS SP8, Leica, Germany). Fluorescence images were acquired with excitation at $488 \mathrm{~nm}$, and the corresponding emissions were detected at 500 and $635 \mathrm{~nm}$, respectively.

\section{Crystal Violet Assay}

Static biofilm formed and processed as described above was carefully washed three times with PBS (Jurcisek et al., 2011; Duncan et al., 2015). The biofilm was heat-fixed at $60^{\circ} \mathrm{C}$ for $1 \mathrm{~h}$, stained with crystal violet for $10 \mathrm{~min}$, and then rinsed with Milli-Q water until rinse water was clear. After removing residual fluid, the stained biofilm was photographed or solubilized in the 


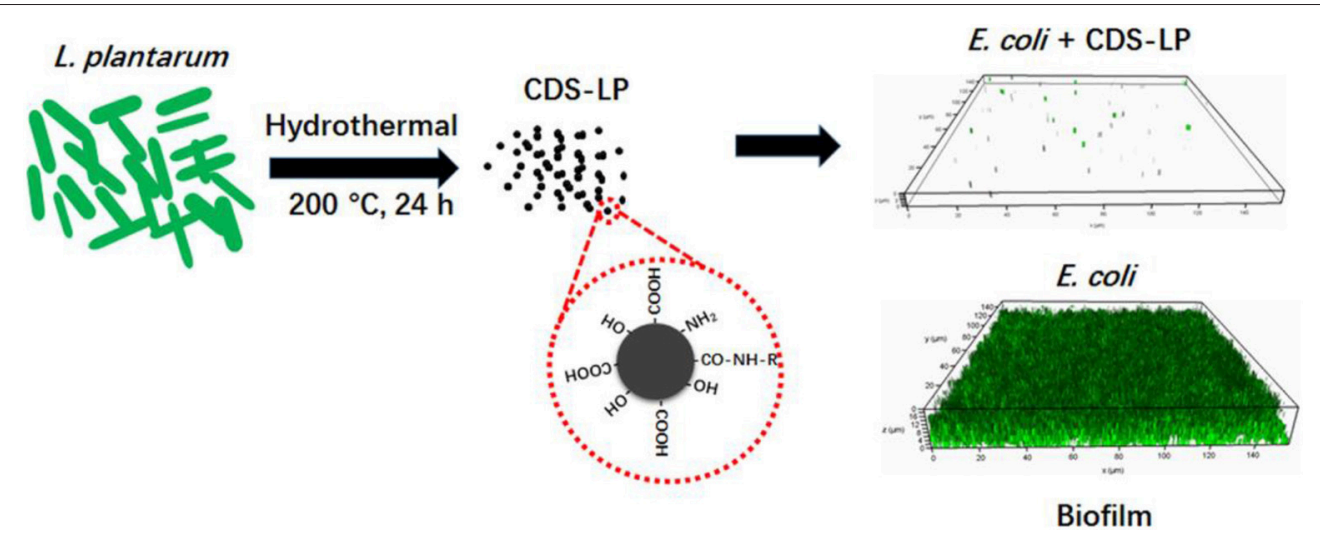

SCHEME 1 | Schematic of the synthetic route and anti-biofilm of CDS-LP.

destaining solution ( $10 \%$ methanol and $7.5 \%$ acetic acid in water). The absorbance at $595 \mathrm{~nm}$ was read on a microplate reader (Multiskan, Thermo Scientific). The experiments were carried out with eight biological replicates.

\section{Effect of CDs-LP on E. coli Growth}

Bacteria were cultured overnight in $\mathrm{LB}$ at $37^{\circ} \mathrm{C}$ with $180 \mathrm{rpm}$. The overnight bacterial cell culture was diluted 1:100 in 1/5 lysogeny broth (LB) without and with different concentrations of CDsLP and then inoculated into the 96-well plates (Costar, Corning, USA) with $100 \mathrm{uL} /$ well. The inoculated 96-well plates and dishes were incubated for $24 \mathrm{~h}$ at $28^{\circ} \mathrm{C}$ without shaking. Before measuring the optical density (OD) at $600 \mathrm{~nm}$, the cultured bacteria were well resuspended by pipetting up and down, and then the OD at $600 \mathrm{~nm}$ was recorded.

In addition, colony forming unit (CFU) counting method (plate count method) was utilized to evaluate the cytotoxicity of CDs-LP to E. coli. Briefly, E. coli in log phase were cultured in 1/5 $\mathrm{LB}$ containing $6 \mathrm{mg} / \mathrm{mL}$ CDs-LP at $28^{\circ} \mathrm{C}$ for $24 \mathrm{~h}$. Each culture was diluted with $1 / 5 \mathrm{LB}$ with a dilution factor of $1 \times 10^{5}$. Then the diluted microbial cells were plated in triplicate on LB agar plates and cultured at $37^{\circ} \mathrm{C}$ for $24 \mathrm{~h}$. Finally, the cell colonies of E. coli were counted. Three biological replicates were performed. The cytotoxicity was evaluated by comparing the number of CFUs in treated groups to that in the control group.

\section{MTT Assay}

AT II cells (normal lung cells) were cultured at $37^{\circ} \mathrm{C}$ with $5 \% \mathrm{CO}_{2}$ in DMEM cell media supplemented with $10 \%$ fetal bovine serum. The cells $\left(5 \times 10^{4}\right.$ cells per well $)$ were seeded into a 96-well plate, cultured overnight and then treated with different concentrations of CDs-LP $(0,0.01,0.05,0.1,0.5,1,3$, and $6 \mathrm{mg} / \mathrm{mL}$ ) for $24 \mathrm{~h} .10 \mu \mathrm{L} \mathrm{3-(4,5-dimethylthiazol-2-yl)-2,5-}$ diphenyltetrazolium bromide (MTT, $5 \mathrm{mg} / \mathrm{mL}$ ) was added to each well. After incubation for $4 \mathrm{~h}$, the culture medium in each well was replaced with $150 \mu \mathrm{L}$ dimethyl sulfoxide (DMSO). The absorbance at $492 \mathrm{~nm}$ was recorded by a Multiskan FC microplate photometer (Thermo).

\section{RESULTS AND DISCUSSION \\ Preparation and Characterization of Carbon Dots}

L. plantarum LLC-605 is a highly exopolysaccharide-producing strain (Li et al., 2017), and might contain a great number of carbohydrates, which can serve as abundant precursors for carbon dots formation together with other biological molecules like proteins and peptides in the bacterium. Furthermore, it belongs to lactic acid bacteria that have healthy benefits for humans. Therefore, carbon dots were fabricated using L. plantarum LLC-605 via one-step hydrothermal carbonization, which is denoted as CDs-LP. CDs-LP were quasi-spherical with an average diameter of $3 \mathrm{~nm}$ as demonstrated in the TEM image (Figure 1A). A crystalline structure can be observed in the highresolution image of CDs-LP with the lattice fringe of $0.32 \mathrm{~nm}$, in consistent with the (002) spacing reported for graphitic carbon (Reckmeier et al., 2016) (Figure 1B). CDs-LP could be homogeneously dispersed in aqueous solution and emitted blue fluorescence under $365 \mathrm{~nm}$ UV light irradiation (Figure 1C). Besides, no characteristic absorption peak was found in the UV-vis spectrum of CDs-LP, which was also observed in other carbon nanomaterials (Zhao et al., 2011; Jiang et al., 2017; Lin et al., 2017). The synthesis mechanism of CDs remains obscure, which might involve decomposing and carbonization of stocks, dehydration condensation, nucleation and growth of carbon in the progress of hydrothermal experiments (Reckmeier et al., 2016).

The quantum yield (QY) of CDs-LP was 10.3\%. CDs-LP has a largely negative charge with a zeta potential of $-22 \mathrm{mV}$, which might due to that its raw material L. plantarum LLC605 is negatively charged. The emission intensity of CDs-LP increased gradually from $300 \mathrm{~nm}$ to $380 \mathrm{~nm}$, and then declined from 400 to $520 \mathrm{~nm}$ (Figure 1D). Meanwhile, the emission peaks of CDs-LP gradually red-shifted from 420 to $540 \mathrm{~nm}$ when the excitation wavelength varied from 300 to $520 \mathrm{~nm}$ (Figure 1D). The maximum emission peak was at $450 \mathrm{~nm}$ with the excitation wavelength of $380 \mathrm{~nm}$. Obviously, the fluorescence emission spectrum of CDs-LP is dependent on the excitation wavelength. 
A

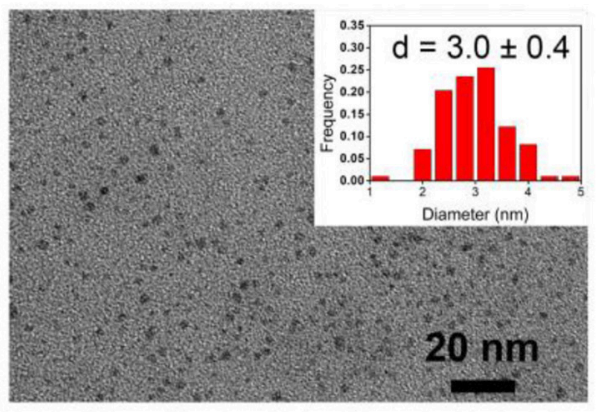

B

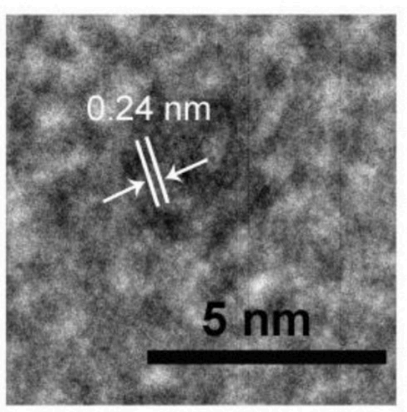

C

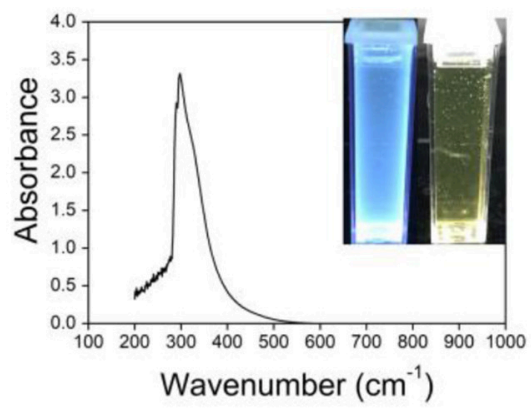

F

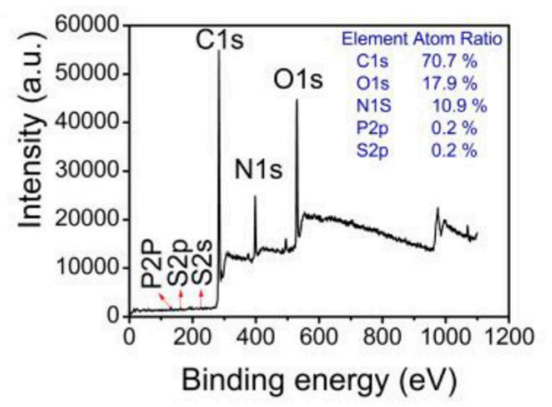

I

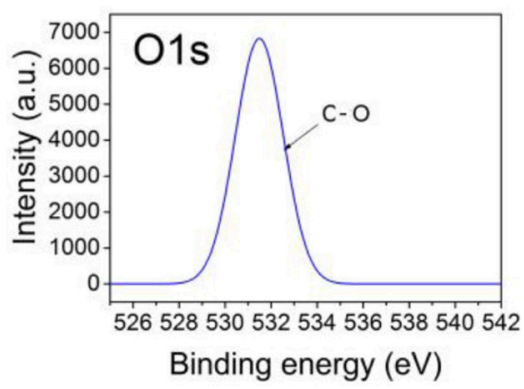

FIGURE 1 | Characterization of CDs-LP. (A) TEM image and the corresponding size distribution histogram of CDs-LP (inset). (B) The high-resolution TEM image of CDs-LP. (C) UV-vis absorption spectra of CDs-LP in water. The insets are photographs of CDs-LP in water with and without irradiation under a 365 nm UV lamp. (D) Fluorescence spectra of CDs-LP dispersed in water. (E) FTIR spectra of dried CDs-LP. (F) XPS spectrum of dried CDs-LP and the high-resolution XPS peaks of C1s (G), N1s (H) and O1s (I).

Although the mechanism behind the red-shift emission property that harbored by a great number of the reported carbon dots (Wang and Zhou, 2014; Yang et al., 2016; Hua et al., 2017b) is still not clear, it is often associated with surface- or defect states in the amorphous carbon shell of CDs near the Fermi level (Reckmeier et al., 2016; Hua et al., 2017b).

FITR and XPS were utilized to investigate the composition of CDs-LP. As shown in Figure 1E, the broad absorption band around $3,188 \mathrm{~cm}^{-1}$ was due to the stretching vibrations of $\mathrm{O}-\mathrm{H}(\nu(\mathrm{O}-\mathrm{H}))$ and/or N-H $(\nu(\mathrm{N}-\mathrm{H}))$. The peak at $1,674 \mathrm{~cm}^{-1}$ belongs to the $\mathrm{C}=\mathrm{O}$ stretching vibration, $\mathrm{v}(\mathrm{C}=\mathrm{O})$, which may come from the amide bond (-CONH-) or the $-\mathrm{COOH}$ group. The peak at $1,593 \mathrm{~cm}^{-1}$ is attributable to the $\mathrm{N}-\mathrm{H}$ bending vibration $\delta(\mathrm{N}-\mathrm{H})$, owing to the amide bond (-CONH-) or the $\mathrm{NH}_{2}$ group. The peak at $1,405 \mathrm{~cm}^{-1}$ may derive from the $\mathrm{C}-\mathrm{H}$ bending vibration $(\delta(\mathrm{C}-\mathrm{H}))$, and the peak at $1,124 \mathrm{~cm}^{-1}$ can be assigned to the $\mathrm{C}-\mathrm{O}$ stretching vibration $(\mathrm{v}(\mathrm{C}-\mathrm{O}))$. CDs-LP contain the elements of $\mathrm{C}(70.7 \%), \mathrm{O}(17.9 \%), \mathrm{N}(10.9 \%), \mathrm{P}$ $(0.2 \%)$, and $S(0.2 \%)$ as measured by XPS (Figure 1F). Note that the $\mathrm{H}$ element cannot be determined by XPS. The two fitted peaks at 285 and $287.6 \mathrm{eV}$ in the high-resolution C1s spectrum (Figure 1G) can be assigned to carbon-containing groups $\mathrm{C}-\mathrm{C} / \mathrm{C}$ $=\mathrm{C}$ (sp2 carbons) and $\mathrm{C}=\mathrm{O}$, respectively (Bao et al., 2015). The N1s peak at 399.7 and $401.5 \mathrm{eV}$ (Figure 1H) could separately be ascribed to amide nitrogen $\mathrm{C}-\mathrm{N}-\mathrm{C}$ and the amino nitrogen N-H (Yang et al., 2014; Zhang and Chen, 2014), evidencing the presence of both amino and amido groups in CDs-LP as found by FTIR. For the O1s spectrum, the fitted peak at $531.5 \mathrm{eV}$ (Figure 1I) is attributed to the oxygen element in the form of C-O (Zhang and Chen, 2014; Ding et al., 2016). In conclusion, 
the FTIR and XPS results showed that the as-synthesized CDsLP contain the elements $\mathrm{C}, \mathrm{H}, \mathrm{O}, \mathrm{N}, \mathrm{P}$, and $\mathrm{S}$, and the functional groups $-\mathrm{OH},-\mathrm{COOH},-\mathrm{NH}_{2}$, and $-\mathrm{CONH}-$.

\section{CDs-LP Inhibit the Formation of $E$. coli Biofilm}

To observe how CDs-LP affect the formation of E. coli biofilms, various amounts of carbon dots were added into the culture media during biofilm growth and the processes of biofilm formation within $72 \mathrm{~h}$ were monitored by the crystal violet assay (Figure 2). Before being assayed by the crystal violet assay (Figure 2) or checked under confocal microscopy (Figure 3), the samples were washed three times with PBS to remove the planktonic cells. So if the biofilm formation is inhibited and the cells do not attach to the surface, the cells would be washed away, leaving fewer cells on the surface. The biofilm formation was reduced gradually from $100 \%$ to $10.2 \%$ in $24 \mathrm{~h}$, as the CDs$\mathrm{LP}$ concentration increased from 0 to $1 \mathrm{mg} / \mathrm{mL}$ (Figure 2A). When the concentration of CDs-LP was $3 \mathrm{mg} / \mathrm{mL}$ and higher, no E. coli biofilm was formed, showing that CDs-LP could effectively inhibit the biofilm formation of $E$. coli when the concentration is above $3 \mathrm{mg} / \mathrm{mL}$. When the treatment time was increased from 24 to $72 \mathrm{~h}$, the inhibition effect was enhanced slightly (Figure 2A). The mean IC50 value for biofilm inhibition (MBIC50) is defined as the lowest concentration at which at least $50 \%$ reduction in biofilm formation was achieved compared to

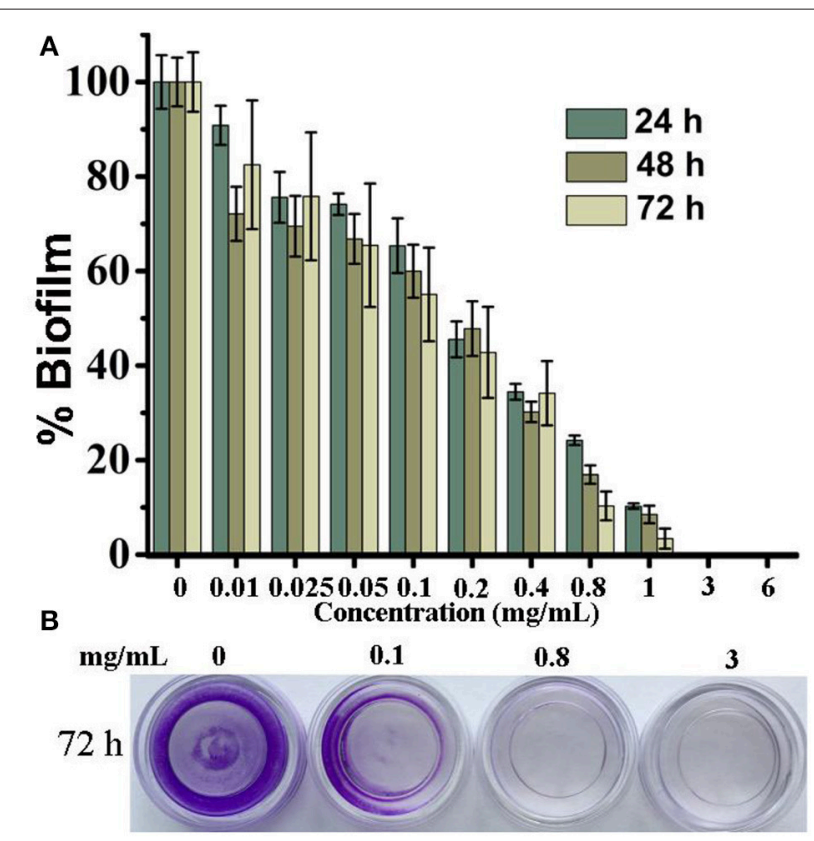

FIGURE 2 | The effect of CDs-LP on the E. coli biofilm was assayed by the crystal violet method. The $E$. coli biofilm was grown at $28^{\circ} \mathrm{C}$ in $1 / 5 \mathrm{LB}$ with the treatment of different concentrations of CDs-LP for different times as indicated in the figure. The absorbance of $E$. coli biofilm in the absence of CDs-LP at 24, 48 , and $72 \mathrm{~h}$ after staining by the crystal violet, was recorded at $595 \mathrm{~nm}$ and arbitrarily assigned as $100 \%$, respectively (A). Also, the E. coli biofilms with the treatment of $0,0.1,0.8$, or $3 \mathrm{mg} / \mathrm{mL}$ CDs-LP for $72 \mathrm{~h}$ were stained by the crystal violet and photographed (B). untreated biofilm (Feldman et al., 2012). Here the MBIC50 of CDs-LP was measured to be $0.4 \mathrm{mg} / \mathrm{mL}$. Moreover, the crystal violet staining results of the $E$. coli biofilms with the treatment of $0,0.1,0.8$, and $3 \mathrm{mg} / \mathrm{mL}$ CDs-LP for $72 \mathrm{~h}$ are shown in Figure 2B. Clearly, the presence of CDs-LP significantly reduced the biofilm formation of E. coli at $0.1 \mathrm{mg} / \mathrm{mL}$ and completely inhibited it at 0.8 or $3 \mathrm{mg} / \mathrm{mL}$, which was in line with the results obtained by measuring the absorbance at $595 \mathrm{~nm}$ (Figure 2A).

Although carbon dots have been successfully exploited in imaging biofilm matrix (Ritenberg et al., 2016) and biofilmencased microorganisms (Lin et al., 2017), it is for the first time to report that carbon dots can inhibit E. coli biofilm formation. Moreover, the synthesis of CDs-LP only requires L. plantarum LLC-605 as the only raw material through one-step hydrothermal reaction, which is very facile and renewable. L. plantarum LLC605 belongs to lactic acid bacteria that have healthy benefits for humans. In biomedical practice, the successful prevention of bacterial biofilm formation could reduce the need for interventional treatments such as systemic and local antibiotic administration, amputation, debridement, or reconstruction, which would improve patient outcomes and reduce the costs associated with infection treatment. In industrial applications, antifouling is important for the performance and durability of the facilities, and cost saving. Therefore, the development of new biofilm inhibitors like CDs-LP here is of great value.

\section{CDs-LP Did Not Inhibit the Growth of $E$. coli}

Confocal microscopy was utilized to evaluate the ability of CDsLP to prevent adhesion and colonization of E. coli. The live and dead bacteria were stained with green and red respectively with the live/dead stain assay (Figure 3). After treatment with CDs-LP of $0.1 \mathrm{mg} / \mathrm{mL}, 0.8 \mathrm{mg} / \mathrm{mL}$, or $3 \mathrm{mg} / \mathrm{mL}$, the $E$. coli biofilm became thinner with a significant reduction in biovolume, as compared to the untreated control. Though several E. coli cells were observed on the culture dish's surface at 0.8 or $3 \mathrm{mg} / \mathrm{mL}$ CDsLP concentration, they are not able to colonize and aggregate to form biofilm. Interestingly, no red fluorescence was observed (Figure 3), showing that there was no dead E. coli cells inside the biofilm and indicating CDs-LP caused no toxicity to E. coli cells. Seemingly, the presence of CDs-LP prevents the colonization and aggregation of $E$. coli on the surface that are prerequisites for E. coli biofilm formation while not killing the cells.

To further confirm that the capability of CDs-LP to inhibit biofilm formation is not due to bactericidal activity, the effect of CDs-LP on the growth of E. coli was tested by both optical density assay and plate count method (Figure 4). The OD of the E. coli culture remained unaltered in the presence of $0-6 \mathrm{mg} / \mathrm{mL}$ CDsLP (Figure 4A). Moreover, the cell viability of $E$. coli incubated with $6 \mathrm{mg} / \mathrm{mL}$ CDs-LP for $24 \mathrm{~h}$ were about 103\% (Figures 4B,C). These results demonstrate that CDs-LP did not block the E. coli growth, which is well correlated with the data obtained by the live/dead assay (Figure 3). Obviously, the anti-E. coli biofilm property of CDs-LP was not attributed to a bactericidal effect, which would be beneficial for the growth of natural bacterial flora. Identification of anti-biofilm compounds that prevent biofilm formation without killing bacteria will provide 


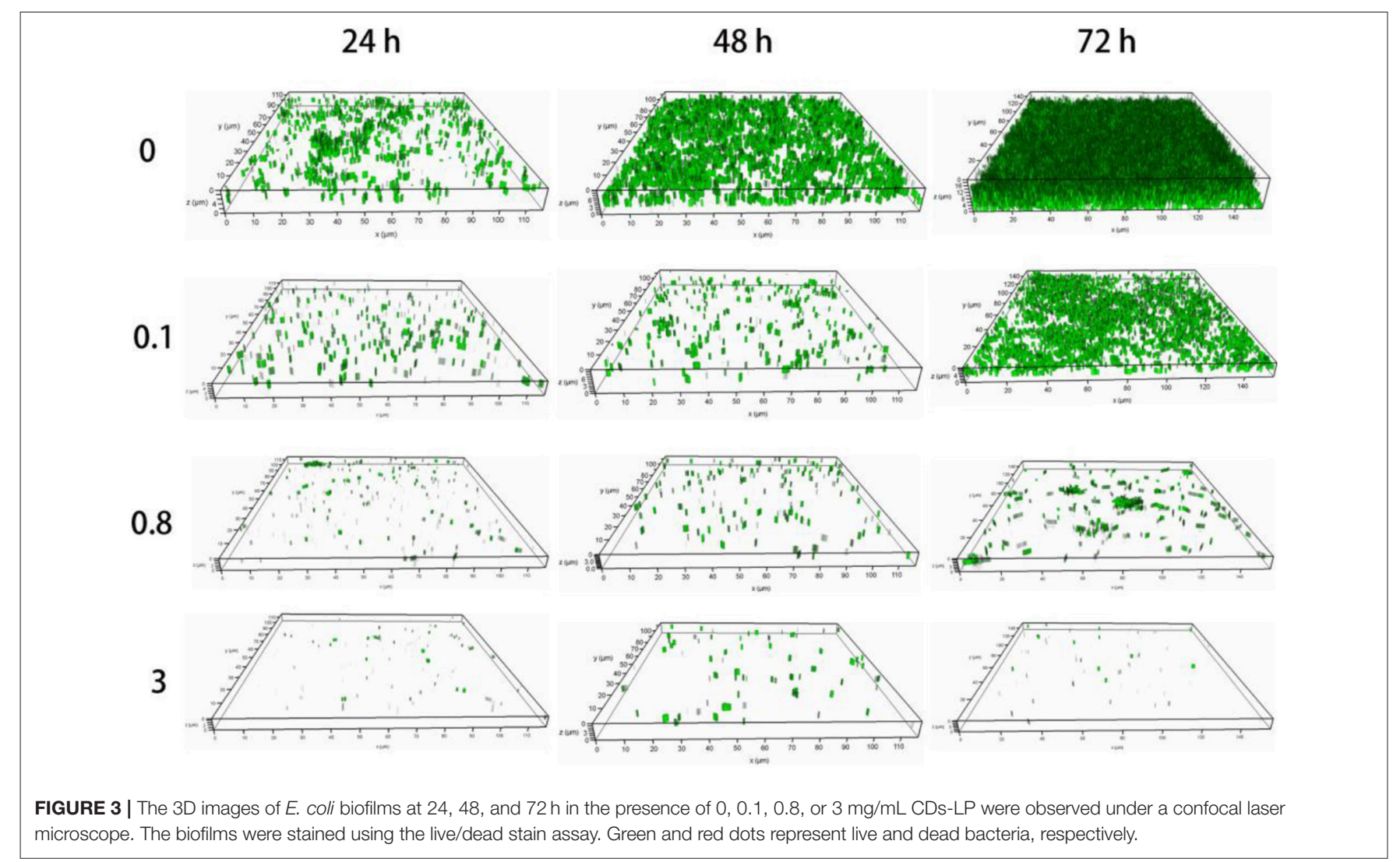

FIGURE 3 | The 3D images of $E$. coli biofilms at 24, 48, and $72 \mathrm{~h}$ in the presence of $0,0.1,0.8$, or $3 \mathrm{mg} / \mathrm{mL}$ CDs-LP were observed under a confocal laser microscope. The biofilms were stained using the live/dead stain assay. Green and red dots represent live and dead bacteria, respectively.
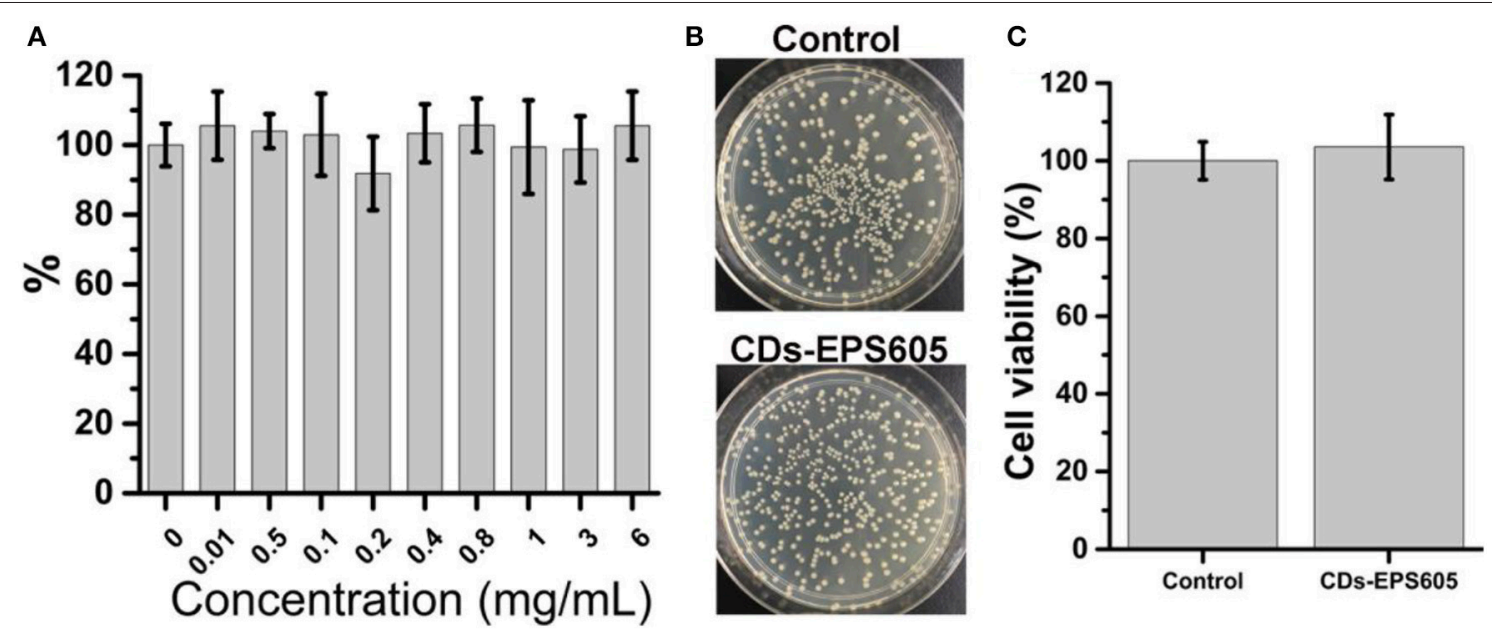

FIGURE 4 | Effect of CDs-LP on E. coli growth. (A) Growth of E. coli for $24 \mathrm{~h}$ in $1 / 5 \mathrm{LB}$ containing 0-6 $\mathrm{mg} / \mathrm{mL} \mathrm{CDs}-\mathrm{LP}$ at $28^{\circ} \mathrm{C}$ without shaking. The absorbance of E. coli cell culture in the absence of CDs-LP was measured at $600 \mathrm{~nm}$ and arbitrarily defined as $100 \%$. (B) Photographs of the agar plates and (C) corresponding statistical histograms of colonies of $E$. coli cultured in $1 / 5$ LB (control) and CDs-LP $(6 \mathrm{mg} / \mathrm{mL}$ ) for $24 \mathrm{~h}$.

a much flexible and practical solution to biofilm infections, because such an approach will not interfere the other noninfectious and natural bacterial flora which are crucial for human and ecosystems. Meanwhile, antifouling coatings nontoxic to marine biota, such as silicone elastomers (Phanindhar et al., 2015) and vanadium pentoxide nanowires (Natalio et al., 2012), have been of great interest, for they do not cause deleterious effects in aquatic environments and are environmentally benign (Lejars et al., 2012). In addition, the non-bactericidal property of CDs-LP implies that the application of CDs-LP in antibiofilm should not lead to antibiotic resistance that long haunts antibiotic-based antibiofilm agents. When it is necessary to 
kill microorganisms, CDs-LP can be simultaneously used in combination with antibiotics. Therefore, preventing E. coli biofilm formation by CDs-LP represents a neutral and flexible strategy.

\section{The CDs-LP Is Non-toxic toward Animal Cells}

To evaluate the safety of CDs-LP, which is important for both biomedical and industrial applications, the relative viabilities of AT II cell with the treatment of CDs-LP in the range from 0 to $6 \mathrm{mg} / \mathrm{mL}$ were measured (Figure 5). More than $80 \%$ of AT II cells remained alive under all the tested concentrations of CDsLP in the range of $0-6 \mathrm{mg} / \mathrm{mL}$, implying that CDs-LP is nontoxic within the concentrations that are utilized to inhibit $E$. coli biofilm formation. The formation of $E$. coli biofilm was inhibited completely by CDs-LP at $1 \mathrm{mg} / \mathrm{mL}$ and higher concentrations. Given that CDs-LP are negatively charged and CDs are well known for their excellent biocompatibility, it is reasonable that CDs-LP did not affect the growth and viability of neither bacteria nor the animal cells.

Several biofilm inhibitors have been reported to be nontoxic to $E$. coli and other bacteria as well as animal cells, such as (5Z)-4-bromo-5-(bromomethylene)-3-butyl-2(5H)-furanone (furanone) (Ren et al., 2001), ursolic acid (Ren et al., 2005), 5-fluorouracil(Attila et al., 2009), indole derivatives (Lee et al., 2007, 2011, 2012) and cationic pillararenes (Joseph et al., 2016). They inhibit the biofilm formation through cell signaling, sulfur metabolism (Wood, 2009) or cysteine metabolism (Lee et al., 2007). Most of these biofilm inhibitors are natural compounds that are not easy to obtain due to scarce resources, while others are synthesized with complicated chemical/physical methods that are not easy to scale up. Contrarily, here the synthesis of CDs-LP

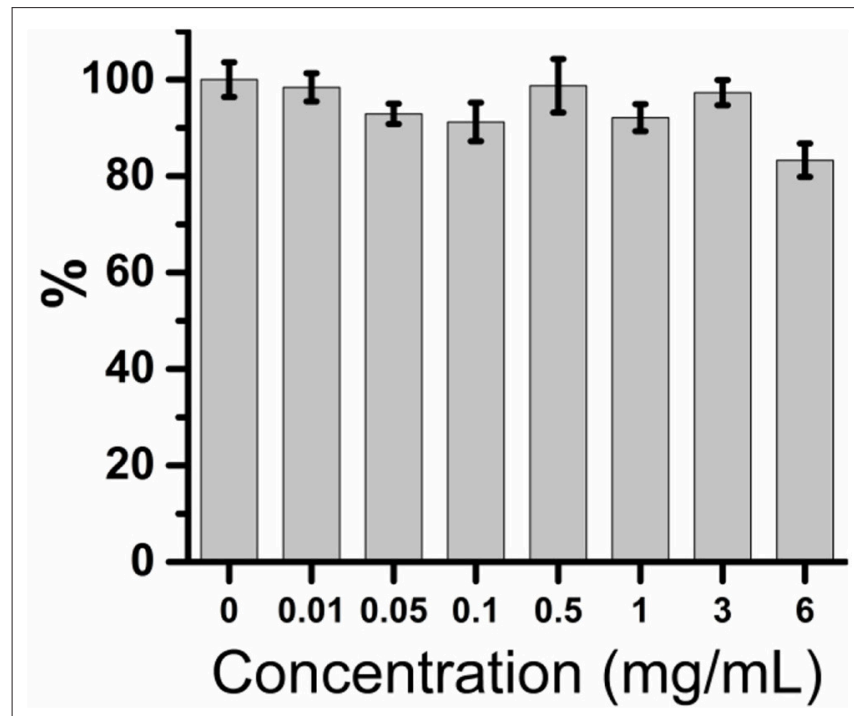

FIGURE 5 | In vitro safety testing result of CDs-LP. Relative viabilities of AT ॥ cell exposed to different concentrations of CDs-LP were determined by MTT assay. The absorbance of AT II cells in the absence of CDs-LP was measured at $492 \mathrm{~nm}$ and arbitrarily assigned as 100\%. is simple, fast and in large amount using the raw material strain LCC605 that is easily accessible. Furthermore, there are active functional groups on CDs-LP surfaces as detected by FTIR and XPS, allowing for further surface engineering to achieve desirable surface coating and bactericidal property.

Most of the current anti-biofilm nanomaterials exhibit potential cytotoxicity and poor biocompatibility, which is mainly caused by the positive charge of cationic nanomaterials that can interact with the negatively charged bacteria/mammalian cell surface through electrostatic interaction. Surface engineering has been often explored to solve this issue. For example, carbon nano/microspheres have been combined with metal nanoparticles to reduce their toxicity and improve their stability (Cui et al., 2012; Li et al., 2014; Cheng et al., 2015). The surface charge of gold nanoparticles was modulated to achieve specific toxicity toward bacterial biofilms while minimizing mammalian cytotoxicity (Giri et al., 2015). Healthy tissues under generally physiological conditions have a $\mathrm{pH}$ of $\sim 7.4$, whereas biofilm is acidic with a $\mathrm{pH}$ of $\sim 5.5$. Therefore nanoparticles were modified with $\mathrm{pH}$-responsive moieties that enable specific target of biofilm and/or drug release at acidic $\mathrm{pH}$ found within biofilm, leading to highly efficient antibiofilm activity without damaging the tissues around biofilm (Horev et al., 2015; Liu et al., 2016; $\mathrm{Hu}$ et al., 2017). Clearly, these surface engineering strategies usually involved complicated chemical/physical modifications. Conversely, the one-step hydrothermal synthesis of CDs-LP reported here is very simple. Given that the most-explored metalcontaining nanomaterials for biofilm treatment have potential cytotoxicity and poor biocompatibility, it is of great importance to develop the metal-free, carbon-based materials that are less toxic and more biocompatible, as demonstrated by CDs-LP in our study.

CDs-LP did not show bactericidal activity toward E. coli, but it could significantly reduce the colonization and aggregation of $E$. coli on the surface that are essential for E. coli biofilm formation. Instead of preventing biofilm formation through disrupting cell membranes and killing bacteria by many nanomaterials which might cause cytotoxicity (Cui et al., 2012; Li et al., 2014; Cheng et al., 2015; Giri et al., 2015; Horev et al., 2015; Liu et al., 2016; Hu et al., 2017), here CDs-LP likely inhibit the biofilm formation through cell signaling (Wood, 2009), sulfur metabolism(Wood, 2009) or cysteine metabolism (Lee et al., 2007), as found for other non-toxic biofilm inhibitors. However, it is not unclear how and which signal pathways in E. coli are affected by CDs-LP and involved in the biofilm inhibition. Further detailed study is required but beyond the scope of this research.

\section{CONCLUSION}

In this study, a new type of carbon dots, CDs-LP, was synthesized by one-step hydrothermal carbonization of L. plantarum LLC605 , which is facile, fast, cheap, and green. CDs-LP are capable of inhibiting E. coli biofilm formation. Most importantly, CDsLP did not inhibit the growth of E. coli and mammalian cells, preventing the generation of bacteria drug-resistance and 
presenting great biocompatibility. CDs-LP represent a novel kind of non-toxic anti-biofilm materials to circumvent the cytotoxicity faced by many current anti-biofilm agents, holding great promise for eco-friendly applications against E. coli biofilm formation for both microbial infection treatment and anti-biofouling material development.

\section{AUTHOR CONTRIBUTIONS}

FL conceived and designed the study. FL carried out the majority of the experiments. CL conducted the characterization of carbon

\section{REFERENCES}

Anderson, G. G., Palermo, J. J., Schilling, J. D., Roth, R., Heuser, J., and Hultgren, S. J. (2003). Intracellular bacterial biofilm-like pods in urinary tract infections. Science 301, 105-107. doi: 10.1126/science. 1084550

Attila, C., Ueda, A., and Wood, T. K. (2009). 5-Fluorouracil reduces biofilm formation in Escherichia coli K-12 through global regulator AriR as an antivirulence compound. Appl. Microbiol. Biotechnol. 82, 525-533. doi: 10.1007/s00253-009-1860-8

Bao, L., Liu, C., Zhang, Z. L., and Pang, D. W. (2015). Photoluminescence-tunable carbon nanodots: surface-state energy-gap tuning. Adv. Mater. Weinheim. 27, 1663-1667. doi: 10.1002/adma.201405070

Boda, S. K., Broda, J., Schiefer, F., Weber-Heynemann, J., Hoss, M., Simon, U., et al. (2015). Cytotoxicity of ultrasmall gold nanoparticles on planktonic and biofilm encapsulated Gram-positive Staphylococci. Small 11, 3183. doi: $10.1002 /$ smll.201403014

Chen, Z., Ji, H., Liu, C., Bing, W., Wang, Z., and Qu, X. (2016). A multinuclear metal complex based dnase-mimetic artificial enzyme: matrix cleavage for combating bacterial biofilms. Angew Chem. Int. Ed. 55, 10732-10736. doi: $10.1002 /$ anie. 201605296

Cheng, X., Fu, A., Li, H., Wang, Y., Guo, P., Liu, J., et al. (2015). Sustainable preparation of copper particles decorated carbon microspheres and studies on their bactericidal activity and catalytic properties. ACS Sust. Chem. Eng. 3, 2414-2422. doi: 10.1021/acssuschemeng.5b00382

Cui, J., Hu, C., Yang, Y., Wu, Y., Yang, L., Wang, Y., et al. (2012). Facile fabrication of carbonaceous nanospheres loaded with silver nanoparticles as antibacterial materials. J. Mater. Chem. 22, 8121-8126. doi: 10.1039/c2jm1 $6441 \mathrm{~h}$

de la Fuente-Núñez, C., Reffuveille, F., Fernandez, L., and Hancock, R. E. (2013). Bacterial biofilm development as a multicellular adaptation: antibiotic resistance and new therapeutic strategies. Curr. Opin. Microbiol. 16, 580-589. doi: 10.1016/j.mib.2013.06.013

Ding, H., Yu, S. B., Wei, J. S., and Xiong, H. M. (2016). Full-color light-emitting carbon dots with a surface-state-controlled luminescence mechanism. ACS Nano 10, 484-491. doi: 10.1021/acsnano.5b05406

Duncan, B., Li, X., Landis, R. F., Kim, S. T., Gupta, A., Wang, L. S., et al. (2015). Nanoparticle-stabilized capsules for the treatment of bacterial biofilms. ACS Nano 9, 7775-7782. doi: 10.1021/acsnano.5b01696

Duong, H. T., Jung, K., Kutty, S. K., Agustina, S., Adnan, N. N., Basuki, J. S., et al. (2014). Nanoparticle (star polymer) delivery of nitric oxide effectively negates Pseudomonas aeruginosa biofilm formation. Biomacromolecules 15, 2583-2589. doi: $10.1021 / \mathrm{bm} 500422 \mathrm{v}$

Feldman, M., Tanabe, S., Howell, A., and Grenier, D. (2012). Cranberry proanthocyanidins inhibit the adherence properties of Candida albicans and cytokine secretion by oral epithelial cells. BMC Complement. Altern. Med. 12:6. doi: $10.1186 / 1472-6882-12-6$

Fischbach, M. A., and Walsh, C. T. (2009). Antibiotics for emerging pathogens. Science 325, 1089. doi: 10.1126/science. 1176667

Flemming, H. C., Wingender, J., Szewzyk, U., Steinberg, P., Rice, S. A., and Kjelleberg, S. (2016). Biofilms: an emergent form of bacterial life. Nat. Rev. Microbiol. 14, 563-575. doi: 10.1038/nrmicro.2016.94 dots. FL and ZC analyzed the data and drafted the manuscript. All authors read and approved the final manuscript.

\section{ACKNOWLEDGMENTS}

This work was supported by the National Natural Science Foundation of China (31700040), the Fundamental Research Funds for the Central Universities and a project funded by the Priority Academic Program Development (PAPD) of Jiangsu Higher Education Institutions. ZC acknowledges the support from University of Michigan.

Gao, W., Vecchio, D., Li, J., Zhu, J., Zhang, Q., Fu, V., et al. (2014). Hydrogel containing nanoparticle-stabilized liposomes for topical antimicrobial delivery. ACS Nano 8, 2900-2907. doi: 10.1021/nn500110a

Geilich, B. M., Gelfat, I., Sridhar, S., van de Ven, A. L., and Webster, T. J. (2017). Superparamagnetic iron oxide-encapsulating polymersome nanocarriers for biofilm eradication. Biomaterials 119, 78-85. doi: 10.1016/j.biomaterials.2016.12.011

Giri, K., Yepes, L. R., Duncan, B., Parameswaran, P. K., Yan, B., Jiang, Y., et al. (2015). Targeting bacterial biofilms via surface engineering of gold nanoparticles. RSC Adv. 5, 105551-105559. doi: 10.1039/C5RA16305F

Horev, B., Klein, M. I., Hwang, G., Li, Y., Kim, D., Koo, H., et al. (2015). pHactivated nanoparticles for controlled topical delivery of farnesol to disrupt oral biofilm virulence. ACS Nano 9, 2390-2404. doi: 10.1021/nn507170s

Hu, D., Li, H., Wang, B., Ye, Z., Lei, W., Jia, F., et al. (2017). Surface-adaptive gold nanoparticles with efective adherence and enhanced photothermal ablation of methicillin-resistant Staphylococcus aureus biofilm. ACS Nano. 11, 9330-9339. doi: 10.1021/acsnano.7b04731

Hua, X. W., Bao, Y. W., Chen, Z., and Wu, F. G. (2017a). Carbon quantum dots with intrinsic mitochondrial targeting ability for mitochondria-based theranostics. Nanoscale 9, 10948-10960. doi: 10.1039/C7NR03658B

Hua, X. W., Bao, Y. W., Wang, H. Y., Chen, Z., and Wu, F. G. (2017b). Bacteria-derived fluorescent carbon dots for microbial live/dead differentiation. Nanoscale, 9, 2150-2161. doi: 10.1039/C6NR06558A

Jiang, Y. W., Gao, G., Zhang, X., Jia, H. R., and Wu, F. G. (2017). Antimicrobial carbon nanospheres. Nanoscale 9, 15786-15795. doi: 10.1039/C7NR0 $4679 \mathrm{~K}$

Joseph, R., Naugolny, A., Feldman, M., Herzog, I. M., Fridman, M., and Cohen, Y. (2016). Cationic pillararenes potently inhibit biofilm formation without affecting bacterial growth and viability. J. Am. Chem. Soc. 138, 754. doi: 10.1021/jacs.5b11834

Jurcisek, J. A., Dickson, A. C., Bruggeman, M. E., and Bakaletz, L. O. (2011). In vitro biofilm formation in an 8-well chamber slide. J. Vis. Exp. 2011:2481. doi: $10.3791 / 2481$

Kostakioti, M., Hadjifrangiskou, M., and Hultgren, S. J. (2013). Bacterial biofilms: development, dispersal, and therapeutic strategies in the dawn of the postantibiotic era. Cold Spring Harb. Perspect. Med. 3:a010306. doi: $10.1101 /$ cshperspect.a010306

Lee, J. H., Cho, M. H., and Lee, J. (2011). 3-indolylacetonitrile decreases Escherichia coli O157:H7 biofilm formation and Pseudomonas aeruginosa virulence. Environ. Microbiol. 13, 62-73. doi: 10.1111/j.1462-2920.2010.02308.x

Lee, J. H., Kim, Y. G., Kim, C. J., Lee, J. C., Cho, M. H., and Lee, J. (2012). Indole-3-acetaldehyde from Rhodococcus sp. BFI 332 inhibits Escherichia coli O157:H7 biofilm formation. Appl. Microbiol. Biotechnol. 96, 1071-1078. doi: 10.1007/s00253-012-3881-y

Lee, J., Bansal, T., Jayaraman, A., Bentley, W. E., and Wood, T. K. (2007). Enterohemorrhagic Escherichia coli biofilms are inhibited by 7 hydroxyindole and stimulated by isatin. Appl. Environ. Microbiol. 73, 4100-4109. doi: 10.1128/AEM.00360-07

Lejars, M., Margaillan, A., and Bressy, C. (2012). Fouling release coatings: a nontoxic alternative to biocidal antifouling coatings. Chem. Rev. 112, 4347-4390. doi: 10.1021/cr200350v 
Li, C., Zhou, L., Yang, H., Lv, R., Tian, P., Li, X., et al. (2017). Self-assembled exopolysaccharide nanoparticles for bioremediation and green synthesis of noble metal nanoparticles. ACS Appl. Mater. Interfaces. 9, 22808-22818, doi: 10.1021/acsami.7b02908

Li, H. T., Kang, Z. H., Liu, Y., and Lee, S. T. (2012). Carbon nanodots: synthesis, properties and applications. J. Mater. Chem. 22, 24230-24253. doi: $10.1039 / \mathrm{c} 2 \mathrm{jm} 34690 \mathrm{~g}$

Li, S., Yan, X., Yang, Z., Yang, Y., Liu, X., and Zou, J. (2014). Preparation and antibacterial property of silver decorated carbon microspheres. Appl. Surf. Sci. 292, 480-487. doi: 10.1016/j.apsusc.2013.11.166

Lin, F., Li, C., Dong, L., Fu, D., and Chen, Z. (2017). Imaging biofilm-encased microorganisms using carbon dots derived from L. plantarum. Nanoscale. 9, 9056-9064. doi: 10.1039/C7NR01975K

Liu, Y., Busscher, H. J., Zhao, B., Li, Y., Zhang, Z., van der Mei, H. C., et al. (2016). Surface-adaptive, antimicrobially loaded, micellar nanocarriers with enhanced penetration and killing efficiency in Staphylococcal biofilms. ACS Nano 10, 4779-4789. doi: 10.1021/acsnano.6b01370

Nandi, S., Malishev, R., Parambath Kootery, K., Mirsky, Y., Kolusheva, S., and Jelinek, R. (2014). Membrane analysis with amphiphilic carbon dots. Chem. Commun. 50, 10299-10302. doi: 10.1039/C4CC03504F

Nandi, S., Ritenberg, M., and Jelinek, R. (2015). Bacterial detection with amphiphilic carbon dots. Analyst 140, 4232-4237. doi: 10.1039/C5AN00471C

Natalio, F., Andre, R., Hartog, A. F., Stoll, B., Jochum, K. P., Wever, R., et al. (2012). Vanadium pentoxide nanoparticles mimic vanadium haloperoxidases and thwart biofilm formation. Nat. Nanotechnol. 7, 530-535. doi: $10.1038 /$ nnano. 2012.91

Nguyen, T. K., Duong, H. T., Selvanayagam, R., Boyer, C., and Barraud, N. (2015). Iron oxide nanoparticle-mediated hyperthermia stimulates dispersal in bacterial biofilms and enhances antibiotic efficacy. Sci. Rep. 5:18385. doi: $10.1038 /$ srep 18385

Nguyen, T. K., Selvanayagam, R., Ho, K. K. K., Chen, R., Kutty, S. K., Rice, S. A., et al. (2016). Co-delivery of nitric oxide and antibiotic using polymeric nanoparticles. Chem. Sci. 7, 1016-1027. doi: 10.1039/C5SC02769A

Shivapooja, P., Yu, Q., Orihuela, B., Mays, R., Rittschof, D., Genzer, J., et al. (2015). Modification of silicone elastomer surfaces with zwitterionic polymers: short-term fouling resistance and triggered biofouling release. ACS Appl. Mater. Interfaces 7, 25586. doi: 10.1021/acsami.5b09199

Reckmeier, C. J., Schneider, J., Susha, A. S., and Rogach, A. L. (2016). Luminescent colloidal carbon dots: optical properties and effects of doping [Invited]. Opt. Express 24, A312-A340. doi: 10.1364/OE.24.00A312

Ren, D., Sims, J. J., and Wood, T. K. (2001). Inhibition of biofilm formation and swarming of Escherichia coli by (5Z)-4-bromo-5(bromomethylene)-3-butyl-2(5H)-furanone. Environ. Microbiol. 3, 731-736. doi: 10.1046/j.1462-2920.2001.00249.x

Ren, D., Zuo, R., Gonzalez Barrios, A. F., Bedzyk, L. A., Eldridge, G. R., Pasmore, M. E., et al. (2005). Differential gene expression for investigation of Escherichia coli biofilm inhibition by plant extract ursolic acid. Appl. Environ. Microbiol. 71, 4022-4034. doi: 10.1128/AEM.71.7.4022-4034.2005

Ritenberg, M., Nandi, S., Kolusheva, S., Dandela, R., Meijler, M. M., and Jelinek, R. (2016). Imaging Pseudomonas aeruginosa biofilm extracellular polymer scaffolds with amphiphilic carbon dots. ACS Chem. Biol. 11, 1265-1270. doi: 10.1021/acschembio.5b01000
Robinson, A. M., Bannister, M., Creeth, J. E., and Jones, M. N. (2001) The interaction of phospholipid liposomes with mixed bacterial biofilms and their use in the delivery of bactericide. Colloids Surf. A 186, 43-53. doi: 10.1016/S0927-7757(01)00481-2

Taglietti, A., Arciola, C. R., D’agostino, A., Dacarro, G., Montanaro, L., Campoccia, D., et al. (2014). Antibiofilm activity of a monolayer of silver nanoparticles anchored to an amino-silanized glass surface. Biomaterials 35, 1779-1788. doi: 10.1016/j.biomaterials.2013.11.047

Wang, L., and Zhou, H. S. (2014). Green synthesis of luminescent nitrogen-doped carbon dots from milk and its imaging application. Anal. Chem. 86, 8902-8905. doi: $10.1021 /$ ac502646x

Wang, Y., and Hu, A. (2014). Carbon quantum dots: synthesis, properties and applications. J. Mater. Chem. C 2, 6921-6939. doi: 10.1039/C4TC0 $0988 \mathrm{~F}$

Wood, T. K. (2009). Insights on Escherichia coli biofilm formation and inhibition from whole-transcriptome profiling. Environ. Microbiol. 11, 1-15. doi: 10.1111/j.1462-2920.2008.01768.x

Yang, J., Zhang, X., Ma, Y. H., Gao, G., Chen, X., Jia, H. R., et al. (2016). Carbon dot-based platform for simultaneous bacterial distinguishment and antibacterial applications. ACS Appl. Mater. Interfaces 8, 32170-32181. doi: 10.1021/acsami.6b10398

Yang, L., Jiang, W., Qiu, L., Jiang, X., Zuo, D., Wang, D., et al. (2015). One pot synthesis of highly luminescent polyethylene glycol anchored carbon dots functionalized with a nuclear localization signal peptide for cell nucleus imaging. Nanoscale 7, 6104-6113. doi: 10.1039/C5NR01080B

Yang, X., Luo, Y., Zhu, S., Feng, Y., Zhuo, Y., and Dou, Y. (2014). One-pot synthesis of high fluorescent carbon nanoparticles and their applications as probes for detection of tetracyclines. Biosens. Bioelectron. 56, 6-11. doi: 10.1016/j.bios.2013.12.064

Zhang, R., and Chen, W. (2014). Nitrogen-doped carbon quantum dots: facile synthesis and application as a "turn-off" fluorescent probe for detection of $\mathrm{Hg}^{2+}$ ions. Biosens. Bioelectron. 55, 83-90. doi: 10.1016/j.bios.2013. 11.074

Zhang, Y., Shen, Y., Teng, X., Yan, M., Bi, H., and Morais, P. C. (2015). Mitochondria-targeting nanoplatform with fluorescent carbon dots for long time imaging and magnetic field-enhanced cellular uptake. ACS Appl. Mater. Interfaces 7, 10201-10212. doi: 10.1021/acsami.5b00405

Zhao, H. X., Liu, L. Q., Liu, Z. D., Wang, Y., Zhao, X. J., and Huang, C. Z. (2011). Highly selective detection of phosphate in very complicated matrixes with an off-on fluorescent probe of europium-adjusted carbon dots. Chem. Comm. 47, 2604-2606. doi: 10.1039/c0cc04399k

Conflict of Interest Statement: The authors declare that the research was conducted in the absence of any commercial or financial relationships that could be construed as a potential conflict of interest.

Copyright (c) $2018 \mathrm{Lin}, \mathrm{Li}$ and Chen. This is an open-access article distributed under the terms of the Creative Commons Attribution License (CC BY). The use, distribution or reproduction in other forums is permitted, provided the original author(s) and the copyright owner are credited and that the original publication in this journal is cited, in accordance with accepted academic practice. No use distribution or reproduction is permitted which does not comply with these terms. 